\title{
Distributed Maintenance of Resource Efficient Wireless Network Topologies (Extended Abstract)
}

\author{
Matthias Grünewald ${ }^{\star}$, Tamás Lukovszki ${ }^{\star \star}$, \\ Christian Schindelhauer ${ }^{\star \star}$, and Klaus Volbert ${ }^{\star \star}$ \\ Heinz Nixdorf Institute, Paderborn University
}

\begin{abstract}
Multiple hop routing in mobile ad hoc networks can minimize energy consumption and increase data throughput. Yet, the problem of radio interferences remains. However if the routes are restricted to a basic network based on local neighborhoods, these interferences can be reduced such that standard routing algorithms can be applied.

We compare different network topologies for these basic networks with respect to degree, spanner-properties, radio interferences, energy, and congestion, i.e. the Yao-graph (aka. $\Theta$-graph) and some also known related models, which will be called the SymmY-graph (aka. YS-graph), the SparsY-graph (aka.YY-graph) and the BoundY-graph. Further, we present a promising network topology called the HL-graph (based on Hierarchical Layers).

Further, we compare the ability of these topologies to handle dynamic changes of the network when radio stations appear and disappear. For this we measure the number of involved radio stations and present distributed algorithms for repairing the network structure.
\end{abstract}

\section{Motivation}

Our research aims at the implementation of a mobile ad hoc network based on distributed robust communication protocols. Besides the traditional use of omni-directional transmitters, we want to investigate the effect of space multiplexing techniques and variable transmission powers on the efficiency and capacity of ad hoc networks. Therefore our radios can send and receive radio signals independently in $k$ sectors of angle $\theta$ using one frequency. Furthermore, our radio stations can regulate its transmission power for each transmitted signal. To show that this approach is also suitable in practical situations, we are currently developing a communication module for the mini robot Khepera [118] that can transmit and receive in eight sectors using infrared light with variable transmission distances up to one meter, see Fig. 1. A colony of Khepera robots will be equipped with

* Dept. of Electrical Engineering and Information Technology, System \& Circuit Technology, gruenewa@hni . uni-paderborn. de. Partially supported by the DFG-SFB 376.

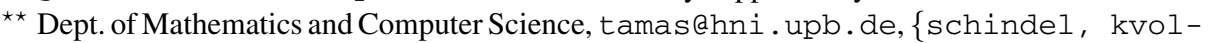
bert $\}$ aupb. de. Partially supported by the DFG-SFB 376 and by the Future and Emerging Technologies programme of the EU, contract nr. IST-1999-14186 (ALCOM-FT). 
this modules to establish ad hoc networks and to evaluate our research results under realistic conditions.

We assume that most of the time the network is stable and performs a point-to-point communication protocol according to an adequately chosen routing protocol. In [10] it is shown that the quality of the routing depends on the choice of the underlying network that we call basic network. In this paper we investigate how such networks can be maintained when stations enter and leave the network.

Little is known about the efficient design of topology-preserving dynamic algorithms. Many approaches consider a model where a central algorithm controls the network structure, using the exact coordinates in $\mathbb{R}^{2}$ of the radio stations (e.g., [3,14]). In contrast to this model we want to investigate a distributed network model where the only information available is given by incoming radio signals and which sector it is received, which gives a rough estimation of the direction to the sender.

The dynamics we are investigating is that a single radio station enters or leaves the system, while the rest of the system is stable. We claim that a node entering a network knows this situation, e.g. because it is switched on or it eavesdrops on existing communication from the network. A node leaving the system is equivalent to a complete node failure. This means that it is not necessary that the leaving node informs the network. Such dynamic changes are the most frequent changes of a radio network besides the motion of radio stations.

In our view its very unlikely that all mobile radio station would start (or leave) at the same time. And even if this is enforced one can easily add a probabilistic strategy that prevents this situation. Then the establishment of the complete network turns out to be a series of single stations entering an existing network. This approach makes sense, since nobody expects that a radio connection to the network is instantly established and we will see that there exist network structures where entering and leaving will only need some logarithmic communication rounds.

In this paper, we do not address the problem of moving radio stations. However, if the movement is not too fast, the moving node can reestablish the correct network by triggering a leave and an enter-operation. Furthermore, we hope that the basic routines developed for this switching dynamics provide basic techniques for more sophisticated maintenance techniques of mobile ad hoc networks.

\section{Model}

Our investigations concentrate on the implementation of distributed algorithms for mobile ad hoc networks with radio stations with specific hardware features. However, some network topologies (like the HL-graph) can be used in a much more general hardware model.

\subsection{Communication Model}

In this paper we assume that if a station enters the system it will send out control messages to stop normal packet routing for the (hopefully short) time needed to update the network structure. All packets are stored on the radio stations and delivered when 
the network structure has been restored. In contrast to this reactive approach, one can also take advantage of synchronized clocks if available. If a periodically time period is reserved that is known to all nodes (including new ones), the maintenance of the network can be done in this special maintenance period. Thus, no control packets are necessary to stop the packet routing mode and collisions caused by the control packets can be prevented.

In our communication model, we assume that a radio station $w$, also called node, is able to detect three types of incoming signals: No signal indicates that no radio signal is transmitted at all or that all radio stations $r$ in distance $d$ send with transmission distance $d^{\prime}<d$. The interference signal indicates that at least two radio stations $u$ and $v$ send in this time step $t$ with transmission distance $d(u, t)>\|u, w\|_{2}$ and $d(v, t)>\|v, w\|_{2}$, where $\|u, w\|_{2}$ denotes the Euclidean distance. A clear signal is received by $w$ if one radio signal with appropriated transmission power to cancel out weaker incoming signals is reaching $u$ 's antenna. Then it can read the transmitted information $m \in\{0,1\}^{p}$ of some length $p$. A communication round is the time necessary to send one packet of length $p$, where $p$ is large enough to carry some elementary information like the sending station, the addressed stations (if specified), the transmission distance, and some control information.

We assume that there is a timing schedule adapted to the basic network topology that allows the stations in a static time period, i.e. no nodes enter or leave, to transmit and acknowledge packets over the network routes with only small number of interfering packets. During such a phase we can neglect the interfering impact of acknowledgment signals.

However when a connection is established the sending and answering signal have the same small length, because only control information needs to be transmitted. Then the impact of answering signals is the same as those of sending signals. Therefore, we consider two types of interferences: The uni-directional interferences in the routing mode and the bi-directional interferences when connections are established or network changes are compensated.

\subsection{Hardware Model}

Every node can choose the transmitting power according to $s$ discrete choices $p_{1}, \ldots, p_{s}$. The energy to send over distance $d$ is given by $\operatorname{pow}(d):=d^{c}$ for some constant $c \geq 2$ (constant factors are omitted for simplicity). This defines the transmitting distances $d_{i}=\left(p_{i}\right)^{1 / c}$ for all $i \in[s]$, where $[s]:=\{1, \ldots, s\}$.

Every node $u$ has $k$ sending and receiving devices, which are located such that they can communicate in parallel within each of $k$ disjoint sectors with angle $\theta=\frac{2 \pi}{k}$. Every node $u$ has been rotated by a angle $\alpha_{u}$, which is unknown to $u$. Note that the radio stations have different offset angles $\alpha_{u}$. If $u$ sends a signal in the $i$ th sector it actually sends into a direction described by the interval $R=\left[\alpha_{u}+i \theta, \alpha_{u}+(i+1) \theta\right)$ and can be received by node $v$ in sector $j$ if $R \cap\left[\alpha_{v}+j \theta, \alpha_{v}+(j+1) \theta\right) \neq \emptyset$. Of course $v$ receives $u$ only if in addition $u$ sends this signal with transmission distance $d_{i} \geq\|u, v\|_{2}$.

Furthermore, we allow that radio stations can measure distances only by sending messages with varying transmission power. Then the receiving party can only decide whether the signal arrives or not. This restricts transmission distances to the set $S=\left\{d_{1}, \ldots, d_{s}\right\}$. 
Define $D: \mathbb{R} \rightarrow\{\varnothing, 1, \ldots, s\}$ as the minimum discrete choice of transmission power to send over a given distance by $D(x):=\min \left\{i \mid d_{i} \geq x\right\}$ if $x \leq d_{s}$ and $D(x):=\varnothing$ if $x>d_{s}$.

Define $\varangle(u, v):=\left\lfloor\frac{\left[\measuredangle(v-u)-\alpha_{u}\right]}{\theta}\right\rfloor \bmod k$ as the number of $u$ 's sector containing the edge $(u, v)$, where $\measuredangle(x)$ denotes the angle of a vector $x$ in $\mathbb{R}^{2}$.

\subsection{Location of Nodes}

One of the most delimiting properties is that radio stations do not know their locations. The following restriction prevent the vertex set from taking abnormal positions.

Definition 1. A vertex set $V$ is in general position, if there are no vertices $u, v, w \in V$ with $v \neq w$ and $\|u, v\|_{2}=\|u, w\|_{2}$. The (Euclidean) distance between two nodes $u, v \in V$ is given by $\|u, v\|_{2}$. We call a vertex set normal, if for a fixed polynomial $p(n)$ we have $\frac{\max _{u, v \in V}\|u, v\|_{2}}{\min _{u, v \in V}\|u, v\|_{2}} \leq p(n)$. We call the locations of radio stations nice, iffor all $u, v, w \in V$ we have $D(u, v) \neq \varnothing$ and $v=w \Longleftrightarrow \varangle(u, v)=\varangle(u, w) \wedge D(u, v)=$ $D(u, w)$.

\section{Basic Network Topologies}

\subsection{Yao-Graphs and Variants}

The underlying hardware model allows to communicate in $k$ disjoint sectors in parallel. Therefore a straight-forward approach is to choose as a communication partner the nearest neighbor in a sector. This leads to the following definition.

Definition 2. The Yao-graph (aka. $\Theta$-graph) is defined by the following set of directed edges: $E:=\{(u, v) \mid \forall w \neq u: \varangle(u, v)=\varangle(u, w) \Rightarrow D(u, v) \leq D(u, w)\}$.

Throughout this paper we assume vertex sets to be nicely located, hence every node has at most one neighbor in a sector. The out-degree is therefore bounded by $k$. However, a node can be the nearest node of many nodes. To overcome this problem of high indegree resulting in time-consuming interference resolution schedules, we present three Yao-graph based topologies.

The symmetric Yao Graph is a straight-forward solution of the high in-degree problem. An edge $(u, v)$ is only introduced if $u$ is the nearest neighbor of $v$ and vice versa.

Definition 3. Let $G_{\theta}$ be the Yao-graph of a vertex set $V$. Then, the Symmetric Yao graph $(\mathbf{S y m m Y})$ is defined by the edge set $E:=\left\{(u, v) \in E\left(G_{\theta}\right) \mid(v, u) \in E\left(G_{\theta}\right)\right\}$.

Although such a graph reduces interferences to a minimum (because in every sector only at most one neighbor appears) very long detours may appear, which make such a graph incapable of bearing short routes and allowing routing without bottlenecks.

Following the approach of [13] we consider also a graph topology which allows at most two neighbors in a sector and call this graph sparsified Yao-graph, which is a Yao-graph where, when the in-degree of a sector exceeds one, only the incoming shortest edge will be chosen. 
Definition 4. For a given vertex set $V$ the edge set of the Sparsified Yao graph (SparsYgraph) is defined by $E:=\left\{(u, v) \in E\left(G_{\theta}\right) \mid \forall w \in V:\left((w, v) \in E\left(G_{\theta}\right)\right.\right.$ and $\varangle(v, w)=$ $\varangle(v, u)) \Rightarrow D(v, w)>D(v, u)\}$, where $G_{\theta}$ denotes the Yao-graph of $V$.

It is an open problem whether all SparsY-graphs are $c$-spanners, i.e. the shortest path between vertices in the network is at most $c$-times longer than the Euclidean distance.

To construct a $c$-spanner with constant degree Arya et al. [2] introduced the following transformation. Like in [9] we apply this technique to the Yao-graph and call the resulting graph a Bounded Degree Yao graph (BoundY graph).

For this, let $G=(V, E)$ be a $c^{\prime}$-spanner with bounded out-degree. Let $N(v)=$ $\{w \in V: w v \in E\}$ the set of in-neighbors of $v \in V$. For each $v \in V$, the star defined by the edges $\{w v \in N(v)\}$ will be replaced by a so-called $v$-single sink $c^{\prime \prime}$-spanner, $c^{\prime \prime}=c / c^{\prime}, T(v)$, which has a bounded in- and out-degree, i.e. $G^{*}=\left(V, E^{*}\right)$, where $E^{*}=\bigcup_{v \in V, u w \in E(T(v))} u w$.

A graph with a vertex set $U$ is called a $v$-single sink $c^{\prime \prime}$-spanner ( $a\left(v, c^{\prime \prime}\right)$-SSS), if from each vertex $w \in U$ there is a $c^{\prime \prime}$-spanner path to the vertex $v$. Such a $\left(v, c^{\prime \prime}\right)$-SSS for $U$ can be constructed as follows.

Let $\alpha=2 \arcsin \frac{c^{\prime \prime}-1}{2 c^{\prime \prime}}$. We divide the plane around $v$ into sectors of an angular diameter at most $\alpha$. For each sector $C$, let $U_{C}$ be the set of all vertices of $U \backslash\{v\}$ contained in $C$. If a subset $U_{C}$ contains more than $|U| / 2$ vertices, then we partition it arbitrarily into two subsets $U_{C, 1} U_{C, 2}$, each of size at most $|U| / 2$ For each subset $U_{C}$, let $w_{c} \in U_{C}$ be the vertex wich is closest to $v$. We add the edge $w_{C} v$ and then we recursively construct a $\left(w_{C}, c^{\prime \prime}\right)$-SSS for each subset $U_{C}$. This recursion ends after $\log |U|$ steps, since we halve (at least) the number of vertices at each level of the recursion. In this way we obtain a directed tree $T(v)$ with root $v$ which is a $\left(v, c^{\prime \prime}\right)$-SSS for $N(v) \cup\{v\}$. Since each vertex $v$ had a bounded out-degree in $G$, and therefore it can be contained in a constant number of in-neighborhoods $N(u), u \in V$, its degree in $G^{*}$ will be also bounded. This completes the construction of the BoundY-graph.

The above recursive construction allows the distributed construction of the BoundY graph given the Yao graph. Furthermore, for compass routing it provides suitable rerouting information: If a message wants to use an edge $u v$ in the Yao-Graph, then it will use the tree-path from $u$ to $v$ in $T(v) \subset G^{*}$, which has at most $O(\log n)$ hops.

\subsection{The Hierarchical Layer Graph}

Adopting ideas from clustering [67] and generalizing an approach of [1] we present a graph consisting of $w$ layers $L_{0}, L_{1}, \ldots, L_{w}$. The union of all this graphs gives the Hierarchical Layer graph (HL graph). The lowest layers $L_{0}$ contains all vertices $V$. The vertex set of a higher layer is a subset of the vertex set of a lower layer until in the highest layer there is only one vertex, i.e. $V=V\left(L_{0}\right) \supseteq V\left(L_{1}\right) \supseteq \cdots \supseteq V\left(L_{w}\right)=$ $\left\{v_{0}\right\}$.

The crucial property of these layers is that in each layer $L_{i}$ vertices obey a minimum distance: $\forall u, v \in V\left(L_{i}\right):\|u, v\|_{2} \geq r_{i}$. Furthermore, all nodes in the next-lower layer must be covered by this distance: $\forall u \in V\left(L_{i}\right) \exists v \in V\left(L_{i+1}\right):\|u, v\|_{2} \leq r_{i+1}$. Our construction uses parameters $\alpha \geq \beta>1$, where for some $r_{0}<\min _{u, v \in V}\|u, v\|_{2}$ we use radii $r_{i}:=\beta^{i} \cdot r_{0}$ and we define in layer $L_{i}$ the edge set $E\left(L_{i}\right)$ by $E\left(L_{i}\right):=$ $\left\{(u, v) \mid u, v \in V\left(L_{i}\right) \wedge\|u, v\|_{2} \leq \alpha \cdot r_{i}\right\}$. 
Clearly, for a normal vertex set we have a maximum number of $w=O(\log n)$ layers. For HL-graphs we need not assume nice or normal locations, as long as our hardware models supports the following transmission distances: $\left\{r_{i}, \alpha r_{i} \mid i \in\{0, \ldots, w\}\right\} \subseteq$ $S=\left\{d_{1}, \ldots, d_{s}\right\}, d_{0} \leq \min _{u, v \in V}\|u, v\|_{2}$ and $d_{w} \geq \max _{u, v \in V}\|u, v\|_{2}$.

\section{Elementary Graph Properties}

We can show the following inclusions. Note that $A \not \# B$ denotes $A \nsubseteq B$ and $B \nsubseteq A$.

Lemma 1. Let $V$ be a nice vertex set. Then, $\operatorname{SymmY}(V) \subseteq \operatorname{Spars} \mathrm{Y}(V) \subseteq \operatorname{BoundY}(V)$ and $\operatorname{Spars} \mathrm{Y}(V) \subseteq \operatorname{Yao}(V)$. For some $V$ it holds that $\operatorname{BoundY}(V) \not \# \operatorname{Yao}(V)$.

\section{Lemma 2.}

For normal and nice vertex sets $V$ consisting of n nodes we observe:

\begin{tabular}{|l|c|c|c|c|c|}
\hline Topology & Yao & SymmY & SparsY & BoundY & $H L$ \\
\hline in-degree & $n-1$ & $k$ & $k$ & $(k+1)^{2}$ & $O(\log n)$ \\
\hline out-degree & $k$ & $k$ & $k$ & $k$ & $O(\log n)$ \\
\hline degree & $n-1$ & $k$ & $2 k$ & $k+(k+1)^{2}$ & $O(\log n)$ \\
\hline
\end{tabular}

Definition 5. A graph $G=(V, E)$ is a c-spanner, if for all $u, v \in V$ there exists a (directed) path p from u to $v$ with $\|p\|_{2} \leq c \cdot\|u, v\|_{2}$. G is a weak $c$-spanner, if for all $u, v \in V$ there exists a path $p$ from $u$ to $v$ which is covered by a disk of radius $c \cdot\|u, v\|_{2}$ centered at $u . G$ is a $(c, d)$-power spanner, iffor all $u, v \in V$ there is a path $p=\left(u=u_{1}, u_{2}, \ldots, u_{m}=v\right)$ from $u$ to $v$ in $G$ such that $\sum_{i=1}^{m-1}\left(\left\|u_{i}, u_{i+1}\right\|_{2}\right)^{d} \leq$ $c \min _{\left(u=v_{1}, v_{2}, \ldots, v_{w}=v\right)} \sum_{i=1}^{w-1}\left(\left\|v_{i}, v_{i+1}\right\|_{2}\right)^{d}$. If for all $d>1$ there exists a constant $c$ such that $G$ is a $(c, d)$-power spanner we call $G$ a power spanner.

On the positive side the following results are known.

Lemma 3. Let $V \subset \mathbb{R}^{2}$.

$-[12]$ For $k>6$ the Yao graph is a c-spanner with $c=1 /\left(1-2 \sin \frac{\theta}{2}\right)$.

- [5] For $k \geq 6$ and $c=\max \left(\left(1+48 \sin ^{4}(\theta / 2)\right)^{1 / 2}, \sqrt{5-\cos \theta}\right)$ the Yao-graph is a weak c-spanner.

- [4] For $k=4$, the Yao-graph is a weak c-spanner with $c=\sqrt{3+\sqrt{5}}$.

- [2] For $k>6$ the BoundY-graph is a c-spanner for a constant $c$.

- [13] For $k>6$ the SparsY-graph is a power spanner

It is an open problem whether SparsY-graphs are $c$-spanners, but we show that they are weak spanners (and the proof of this theorem can be used give a proof of the power spanner property without assuming that the angle $k$ is depending on $V$ as done in [13]). In theorem 3 we prove the open problem stated in [13].

Theorem 1. For $k>6$ the SparsY-graph is a weak c-spanner where $c=\frac{1}{1-2 \sin \frac{\theta}{2}}$.

Proof. Let $G=(V, E)$ be the SparsY-graph and $G_{Y}=\left(V, E_{Y}\right)$ be the underlying Yaograph. Starting from two vertices $u, v$ we will show how to find a directed path from $u$ to $v$ in the SparsY-graph that is inside a disk with center at $u$ of radius $\|u, v\|_{2} /\left(1-2 \sin \frac{\theta}{2}\right)$. For a sector $i$, define the Yao-neighbor $v$ of a vertex $u$ as the (unique) vertex $v$ with $(u, v) \in E_{Y}$. Then we know: 


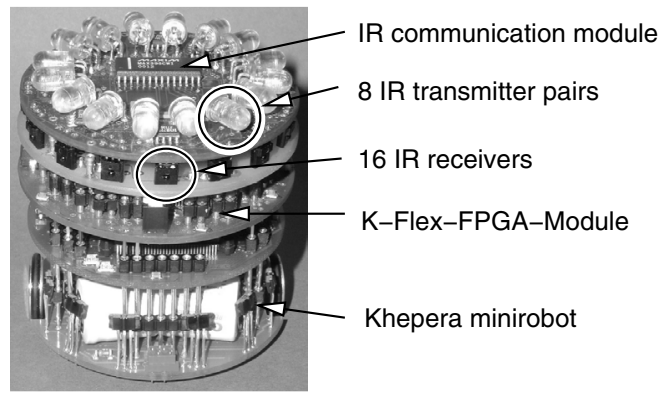

Fig. 1. The mini-robot Khepera equipped with an infrared communication module designed for sectorbased variable-power communication.

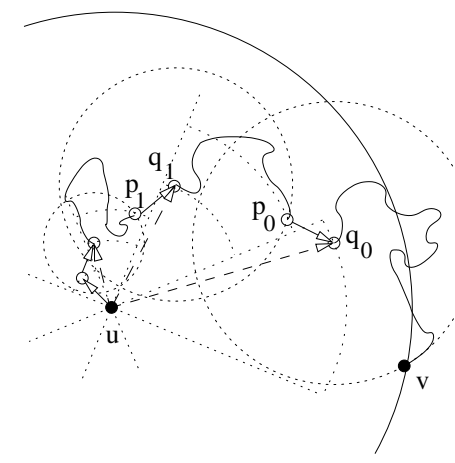

Fig. 2. Weak spanner property of the SparsY-graph

- If a node $u$ has no directed edge in a sector $i$, then either the sector is empty (i.e. no edge in the Yao-graph), or there is a Yao-neighbor $v$ (i.e., $(u, v) \in E_{Y}$ ) incident to an edge $(w, v) \in E$, where $w$ is in another sector of $u$. Furthermore, $\|u, w\|_{2}<\|u, v\|_{2}$, because $\theta<\pi / 3$ and $\|v, w\|_{2}<\|u, v\|_{2}$.

- Every node $u$ has at least one neighbor $v$, i.e. $\exists v \in V:(u, v) \in E$.

Now, we recursively construct the path $P(u, v)$ using some of the Yao-neighbors of $u$ (see Fig. 2). If $(u, v) \in E$ then $P(u, v)=((u, v))$, if $u=v$ then $P(u, v)=()$. If in sector $i=\varangle(u, v)$ the Yao-neighbor, called $q_{0}$, is not directly connected to $u$. Then, we know that there exists an edge $\left(p_{0}, q_{0}\right) \in E$, where $p_{0}$ is in a sector $i_{1} \neq i_{0}$ of $u$ and $\left\|p_{0}, u\right\|_{2}<\left\|q_{0}, u\right\|_{2}$. Furthermore we have that $\left\|q_{0}, u\right\|_{2} \leq\|u, v\|_{2}$. Then, we repeat this consideration for the sector $i_{1}$ and replace $v$ by $p_{1}$. This iteration ends when a Yao-neighbor $q_{m}$ or $p_{m}$ is directly connected to $u$, i.e. $\left(u, q_{m}\right) \in E$ or $\left(u, p_{m}\right) \in E$. Because every node has at least one neighbor in $E$ this process terminates.

Now we recursively define the path $P(u, v)$ from $u$ to $v$ that terminates at node $q_{m}$ (for $p_{m}$ the path can be defined analogous: replace $\left(u, q_{m}\right)$ by $\left(u, p_{m}\right) \circ\left(p_{m}, q_{m}\right)$ ) by

$P(u, v)=\left(u, q_{m}\right) \circ P\left(q_{m}, p_{m-1}\right) \circ\left(p_{m-1}, q_{m-1}\right) \circ \ldots \circ P\left(q_{1}, p_{0}\right) \circ\left(p_{0}, q_{0}\right) \circ P\left(q_{0}, v\right)$.

Note that all nodes $p_{i}, q_{i}$ are inside the disk with center $u$ and radius $\|u, v\|_{2}$. Furthermore, we have $\left\|q_{i}, p_{i-1}\right\|_{2}<\|u, v\|_{2}$. In the next recursion vertices of the path may lie outside of this disk. However it is straight-forward that the maximum disk amplification of a recursion step is $2 \sin \frac{\theta}{2}\|u, v\|_{2}$. That means, that the maximum amplification of the disk with center $u$ and radius $\|u, v\|_{2}$ can be at most $\|u, v\|_{2}+2 \sin \frac{\theta}{2}\|u, v\|_{2}$ in each recursion step. Let $r$ be the depth of the recursion, then by $\sum_{i=0}^{r}\left(2 \sin \frac{\theta}{2}\right)^{i}\|u, v\|_{2} \leq$ $\|u, v\|_{2} /\left(1-2 \sin \frac{\theta}{2}\right)$ it follows, that $P(u, v)$ is inside the disk with center $u$ of radius $\|u, v\|_{2} /\left(1-2 \sin \frac{\theta}{2}\right)$ and so we get $c=1 /\left(1-2 \sin \frac{\theta}{2}\right)$.

Theorem 2. If $\alpha>2 \frac{\beta}{\beta-1}$ the HL-graph is a c-spannerfor $c=\max \left\{\beta \frac{\alpha(\beta-1)+2 \beta}{\alpha(\beta-1)-2 \beta}, \frac{\alpha}{\beta}\right\}$. 
Proof. Define a directed tree $T$ on the vertex set $V_{0} \times\{0, \ldots, w\}$ as follows. The leaves of $T$ are all pairs $V_{0} \times\{0\}$. If $u \in V\left(L_{i}\right)$, then $(u, i)$ is a vertex of $T$. $T$ consists of the following edges: For $i>0$ if $u \in V\left(L_{i}\right)$, then $((u, i-1),(u, i)) \in E(T)$. If $u \in V\left(L_{i}\right) \backslash V\left(L_{i+1}\right)$ then one chooses an arbitrary vertex $v \in V\left(L_{i+1}\right)$ with $(u, v) \in E\left(L_{i}\right)$ and add $((u, i),(v, i+1))$ to the edge set of the tree $T$. Note that this construction describes a tree of depth $w$ and root $\left(v_{0}, w\right)$.

Now for two vertices $u, v \in V$ we define a clamp of height $j$, which is a path connecting $u$ and $v$. The clamp consists of two paths $P_{u}^{j}:=\left(u, p(u), p^{2}(u), \ldots, p^{j}(u)\right)$ and $P_{v}^{j}:=\left(v, p(v), p^{2}(v), \ldots, p^{j}(v)\right)$ of length $j-1$, where $p^{i}(w)$ denotes the ancestor of height $i$ of a vertex $w$ in the tree $T$. These two path are connected by the edge $\left(p^{j}(u), p^{j}(v)\right)$. The following claims now imply the proof. They can be proved by an induction over the number of layers.

Claim. If for vertices $u, v$ the distance is bounded by $\|u, v\|_{2} \leq d_{j}$, then a clamp of height $j$ is contained in the HL-graph, where $d_{0}=\alpha r_{0}$ and $d_{j+1}:=(\alpha \beta-\alpha-2 \beta) r_{j}+d_{j}$.

Claim. A clamp $C$ of height $j$ has maximum length $\ell_{j}$, where $\ell_{0}:=\alpha r_{0}$ and $\ell_{j+1}:=$ $(\alpha \beta-\alpha+2 \beta) r_{j}+\ell_{j}$.

Theorem 3. The SymmY-graph is neither a weak $c$-spanner for any constant $c \in \mathbb{R}$, nor $a(c, d)$-power spanner for any $d>1$.

Proof. We show an example for $n$ points in the plane, such that the SymmY-graph of that points is not a weak $c$-spanner for any $c$. Let $\ell_{1}$ and $\ell_{2}$ be two vertical lines of unit distance from each other, such that $\ell_{2}$ is right to $\ell_{1}$. Rotate $\ell_{1}$ clockwise around its intersection point with the $x$-axis by a very small angle $\delta_{c}$, and rotate $\ell_{2}$ counterclockwise around its intersection point with the $x$-axis by an angle $\delta_{c}$. We denote the rotated lines by $\ell_{1}^{\prime}$ and $\ell_{2}^{\prime}$. Consider the vertex sets $U=\left\{u_{1}, \ldots, u_{m}\right\}$ and $V=\left\{v_{1}, \ldots, v_{m}\right\}, m=n / 2$, placed on $\ell_{1}^{\prime}$ and $\ell_{2}^{\prime}$, respectively, as follows. Assume that for each point $u \in U$, the half-line, halving the $i$ th sector of $u$ is horizontal and directed in positive $x$-direction, and for $v \in V$, the half-line, halving the $i^{\prime}$ th sector of $v$ is horizontal and directed in negative $x$-direction. The vertex $u_{1}$ is placed on the intersection point of $\ell_{1}$ and the $x$-axis. We place $v_{1}$ on $\ell_{2}^{\prime}$ such that $v_{1}$ is in the $i$ th sector of $u_{1}$ and it is very close to the upper boundary of the $i$ th sector of $u_{1}$. The vertex $u_{2}$ is placed on $\ell_{1}^{\prime}$ in the $i^{\prime}$ th sector of $v_{1}$ close to the upper boundary of that sector. The vertex $v_{2}$ is placed on $\ell_{2}^{\prime}$ in the $i$ th sector of $u_{2}$ close to the upper boundary of that sector, etc... Then the SymmY-graph does not contain any edge $(u, v)$ such that $u \in U \backslash\left\{u_{m}\right\}$ and $v \in V \backslash\left\{v_{m}\right\}$. The nearest neighbor of $u_{1}$ in sector $i$ is $v_{1}$, while $v_{1}$ has $u_{1}$ and $u_{2}$ also in sector $i^{\prime}$, where $u_{2}$ is nearer, etc... Only the last link $u_{m}, v_{m}$ will be established. Therefore, even if there is a path from $u_{1}$ to $v_{1}$ in the SymmY-graph, its length is at least $\left\|u_{1}, u_{m}\right\|_{2}+\left\|u_{m}, v_{m}\right\|_{2}+\left\|v_{m}, v_{1}\right\|_{2}$. For any given $c$ we can choose $\delta_{c}$ appropriately small, in order to get $\left\|u_{1}, u_{m}\right\|_{2},\left\|v_{m}, v_{1}\right\|_{2} \geq c / 2$. This proves the claim.

Theorem 4. For $k \geq 6$ and for general vertex sets the SymmY-graph is connected.

Proof. We prove this by an induction over the distance of vertices $\|u, v\|_{2}$. First, note that the closest pair of vertices form an edge of the SymmY-graph. Now observe for two 
vertices $u, v$ : Either there is an edge from $u$ to $v$ or there is a vertex $w$ with $\varangle(u, v)=$ $\varangle(u, w)$ and $\|u, w\|_{2}<\|u, v\|_{2}$ (or symmetrically $\varangle(v, u)=\varangle(v, w)$ and $\|v, w\|_{2}<$ $\|u, v\|_{2}$ ). Because $\theta \leq \pi / 3$ we have $\|v, w\|_{2}<\|u, v\|_{2}$. By induction there is a path from $u$ to $w$ and a path from $w$ to $v$. Therefore a path from $u$ to $v$ exists.

\section{Network Properties}

In [10] we investigate the basic network parameters interference number, energy, and congestion. In this paper we extend the definition of interference number to directed communication. The reason is that we allow two communication modes. In the packet routing mode acknowledgment signals are very short and we can neglect its impact on the interferences. When control messages have to be exchanged sending and answering signals are both short, then we have to consider all combination of interferences. Therefore we distinguish the following of interferences.

Definition 6. The edge $(r, s)$ has a uni-directional interference caused by $(u, v)$, denoted by $(r, s) \in \operatorname{UInt}(u, v)$, if $\varangle(s, r)=\varangle(s, u)$ and $\varangle(u, s)=\varangle(u, v)$ and $D(u, v) \geq D(u, s)$. The edge $(r, s)$ bi-directionally interferes with $(u, v)$, denoted by $(r, s) \in \operatorname{BInt}(u, v)$, if $(r, s) \in \operatorname{UInt}(u, v)$ or $(s, r) \in \operatorname{UInt}(u, v)$ or $(r, s) \in \operatorname{UInt}(v, u)$ or $(s, r) \in \operatorname{UInt}(v, u)$. The (bi-directional) interference number of a basic network $G$ is defined by $\max _{e \in E}\{1+|\operatorname{BInt}(e)|\}$, where $\operatorname{BInt}(e)$ denotes the set of edges that interfere with e if packets are simultaneously transmitted.

Analogously, we define the uni-directional interference number of a graph, by replacing $\operatorname{BInt}(e)$ by $\operatorname{UInt}(e)$.

Note that both types of interferences are asymmetric, i.e. $u \in \operatorname{BInt}(v) \Leftrightarrow v \in$ $\operatorname{BInt}(u)$ and analogously for UInt. This stems from the fact that we use adjustable transmission distances.

A routing protocol can be described by a set of paths $\mathcal{P}$, called path system, that optimizes network parameters. We assume that the path system is chosen according to a demand $w: V \times V \rightarrow \mathbb{N}$ representing the point-to-point communication traffic within the network. Since the locations of the vertex sets are nice for every combination of vertices there is at least a path $p$ from $u$ to $v$ in the path system if $w(u, v)>0$.

Definition 7. The load $\ell(e)$ of an edge $e$ is the number of packets that are using this edge. The interfering load of an edge is $\ell(e)+\sum_{e^{\prime} \in \operatorname{Uint}(e)} \ell\left(e^{\prime}\right)$. The edge with the maximum interfering load defines the congestion of a path system. The energy of a path system $\mathcal{P}$ is given by $\sum_{P \in \mathcal{P}} \sum_{e \in P} \operatorname{pow}(e)$, where $\operatorname{pow}(e)=\left(\|e\|_{2}\right)^{2}$. This is $\sum_{e} \ell(e)\left(\|e\|_{2}\right)^{2}$.

It turns out that energy and congestion are connected to power spanners and weak spanners. The link between these geometric properties and the networking features is described by the following theorem:

Lemma 4. (i) If the basic network is a $(c, d)$ power-spanner, then it allows a path system that approximates the optimal energy path system by a constant factor of $c$.

(ii) Every c spanner is $\left(c^{d}, d\right)$ power-spanner. 
(iii) Iffor a normal vertex set the basic network is a weak c-spanner $G$ with uni-directional interference number $q$ then there is a path system in $G$ that approximates the optimal path system minimizing the congestions by a factor of $O(q \log n)$.

Combining this Lemma with the basic graph properties investigated in section 3 we obtain:

Theorem 5. For a nicely located vertex set $V$ the following table describes the features of our graph topologies.

\begin{tabular}{|r|c|c|c|c|}
\hline Topology & $\begin{array}{c}\text { uni-directional } \\
\text { interference } n \text { r. }\end{array}$ & Spanner & $\begin{array}{c}\text { Energy } \\
\text { approx.factor }\end{array}$ & $\begin{array}{c}\text { Congestion } \\
\text { approx. factor }\end{array}$ \\
\hline Yao-graph & $n-1$ & yes & $O(1)$ & - \\
\hline SymmY-graph & 1 (bi-direct.!) & no, but connected & - & - \\
\hline SparsY-graph & 1 & weak and power spanner & $O(1)$ & $O(\log n)$ \\
\hline BoundY-graph & $\Theta(n)$ & yes & $O(1)$ & - \\
\hline HL-graph & $O(\log n)$ & yes & $O(1)$ & $O\left(\log ^{2} n\right)$ \\
\hline
\end{tabular}

\section{Maintaining the Network}

The standard mode of an ad hoc network is the packet routing mode. In the lucky case of SymmY-graphs there are no interferences between messages and acknowledgments of different edges. For the SparsY-graph packets sent along the direction of the edges cannot interfere with other packets on different edges. However, acknowledgment signals of such edges can interfere. Since in the normal transportation mode data packets are long compared to the short acknowledgments, we neglect this interaction.

In all other graphs we have to resolve (uni-directional) interference. There are two strategies:

Non-interfering Deterministic Schedule. In general it is an $\mathcal{N} \mathcal{P}$-hard problem to compute a schedule that resolves all interferences within optimal time.

However, in the HL-graph in each layer the bi-directional interference number is a constant. Hence, it is easy to define a deterministic schedule that ensures each edge a time frame of $\frac{1}{c \log n}$, which in the worst case slows down communication only by this logarithmic factor.

For the Yao graph the (uni-) directional interferences are given by the in-degree. Hence a straight-forward strategy is to assign each of these incoming senders a time frame of same size. Unlike as for the HL-graph this schedule is far from being optimal, since it does not reflect the actual load on the edges.

The main advantage of such a non-interfering schedule is that collisions immediately indicate that dynamic changes have occurred.

Interfering Probabilistic Schedule. Following the ideas presented in [1] every edge $e$ of the basic network is activated with some independent probability $p(e) \leq \frac{1}{2}$, where for all edges $e$ it holds $p(e)+\sum_{e^{\prime} \in \operatorname{UInt}(e)} p\left(e^{\prime}\right) \leq 1$.

Then, there is a constant probability of at least $\frac{1}{4}$ that a packet is transferred without being interfered by another packet.

The detection of dynamic network changes may need more time than in non-interfering schedules. Here, since with probability of at least $\frac{1}{4}$ every receiver does not get an 
input signal, it suffices to repeat the dynamic change signal for some $O(\log n)$ rounds. Then all nodes are informed with probability $1-1 / p(n)$ (for some polynomial $p(n)$ ).

The only information necessary to maintain such a probabilistic schedule is the local number of uni-directional interferences, or an approximation of that number. In the case of the BoundY-graph this number is not given by a graph property as in the other topologies. Therefore, a node has to inform all $m$ interfering nodes, that they interfere and how many of them interfere. A straight-forward approach shows that this takes time $O(m)$. However we state a general approach that computes and transmits an appropriate approximation of that number in time $O(\log m)$.

We investigate two elementary dynamic operations necessary to maintain dynamic wireless networks:

Enter: While the network is distributing some packets, one radio station wants to enter the network. It will send a special signal causing a special interference signature that will cause all radio station in some specified distance to stop the point-to-point communication mode and switch to a special enter node.

Then, this part of the network devotes its communication to insert the new node into the network topology. After this, it will resume to the normal transportation mode.

Leave: A single station stops sending and receiving. At some time a neighbored node notices this failure and signals it to other nodes of the network. These nodes halt routing packets and rebuild the network.

The two important resources in these update processes are time and number of involved processors. If these parameters are minimized, then the impact of the network disturbance can be kept to a minimum.

Theorem 6. For a normal and nicely located vertex set $V$ the $\Theta(|V|)$ edges need to be changed if an enter/leave operation happens in a Yao-, SymmY-, SparsY-, or BoundYgraph. For the HL-graph this number is bounded by $O(\log |V|)$.

Clearly, this worst case behavior is not the typical situation. Therefore we introduce the number of involved vertices $m$ as an additional parameter into the analysis of the time behavior of the enter/leave algorithms.

Theorem 7. For a normal and nicely located vertex set $V$ and $m$ edges are involved an enter/leave operation can be performed in the Yao based graphs in time $O(m \log s)$. For the HL-graph the time is bounded by $O(\log |V|+\log s)$.

\section{Conclusions}

The following table summarizes the results concerning communication and dynamic performance of the five graph topologies. It turns out that the best dynamic behavior can be achieved by the HL-graph. From the Yao graph variants the SparsY graph outperforms the HL-graph on the approximation factor of congestion. In this overview the SymmYgraph gives the worst impression. Nevertheless, it guarantees that no signals interfere at all. Therefore for a small number of radio stations or average locations it may outperform all the other graph types. 


\begin{tabular}{|r|c|c|c|c|}
\hline Topology & $\begin{array}{c}\text { Congestion } \\
\text { approx. factor }\end{array}$ & $\begin{array}{c}\text { Energy } \\
\text { approx. factor }\end{array}$ & $\begin{array}{c}\text { time for } \\
\text { enter \& leave }\end{array}$ & $\begin{array}{c}\text { enter/leave } \\
\text { involved nodes }\end{array}$ \\
\hline Yao-graph & - & $O(1)$ & $O(n \log s)$ & $\Theta(n)$ \\
\hline SymmY-graph & - & - & $O(n \log s)$ & $\Theta(n)$ \\
\hline SparsY-graph & $O(\log n)$ & $O(1)$ & $O(n \log s)$ & $\Theta(n)$ \\
\hline BoundY-graph & - & $O(1)$ & $O(n \log s)$ & $\Theta(n)$ \\
\hline HL-graph & $O\left(\log ^{2} n\right)$ & $O(1)$ & $O(\log n+\log s)$ & $O(\log n)$ \\
\hline
\end{tabular}

\section{References}

1. M. Adler and Ch. Scheideler. Efficient Communication Strategies for Ad-Hoc Wireless Networks (extended Abstract). In Proc. SPAA'98, pages 259-268, 1998.

2. S. Arya, G. Das, D. M. Mount, J. S. Salowe, and M. H. M. Smid. Euclidean Spanners: Short, Thin, and Lanky. In Proc. STOC, pages 489-498, 1995.

3. B. Chen, K. Jamieson, H. Balakrishnan, and R. Morris. Span: An Energy-Efficient Coordination Algorithm for Topology Maintenance in Ad Hoc Wireless Networks. In Proc. MobiCom, 2001.

4. M. Fischer, T. Lukovszki, and M. Ziegler. Geometric searching in walkthrough animations with weak spanners in real time. In Proc. ESA, pages 163-174, 1998.

5. M. Fischer, F. Meyer auf der Heide, and W.-B. Strothmann. Dynamic data structures for realtime management of large geometric scenes. In Proc. ESA, pages 157-170, 1997.

6. J. Gao, L. J. Guibas, J. Hershberger, L. Zhang, and A. Zhu. Discrete mobile centers. In Proc. Symposium on Computational Geometry, pages 188-196, Medford, MA, USA, 2001.

7. J. Gao, L. J. Guibas, J. Hershberger, L. Zhang, and A. Zhu. Geometric spanner for routing in mobile networks. In Proc. Symposium on Mobile Ad Hoc Networking and Computing, pages 45-55, 2001.

8. K-Team S.A. Khepera miniuature mobile robot, 2000. http://www.k-team.com/robots/khepera.

9. T. Lukovszki. New results on fault tolerant geometric spanners. In Sixth Workshop on Algorithms and Data Structures, pages 193-204, 1999.

10. F. Meyer auf der Heide, Ch. Schindelhauer, K. Volbert, and M. Grünewald. Congestion, Energy and Delay in Radio Networks. To appear at SPAA, 2002.

11. F. Mondada, E. Franzi, and A. Guignard. The development of khepera. In Proc. of the 1st International Khepera Workshop, pages 7-13, Paderborn, Germany, December 10.-11. 1999.

12. J. Ruppert and R. Seidel. Approximating the $d$-dimensional complete Euclidean graph. In 3rd Canadian Conference on Computational Geometry (CCCG '91), pages 207-210, 1991.

13. Y. Wang and X.-Y. Li. Distributed Spanner with Bounded Degree for Wireless Ad Hoc Networks. In Parallel and Distributed Computing Issues in Wireless networks and Mobile Computing, 2002.

14. Y. Xu, J. Heidemann, and D. Estrin. Geography-informed Energy Conservation for Ad Hoc Routing. In Proc. Mobicom, 2001. 